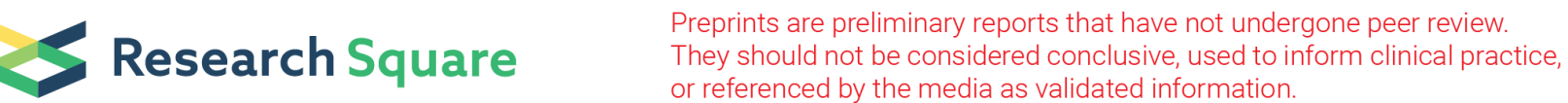

\section{A Positive Feedback Loop Between Gastric Cancer Cells and Tumor-associated Macrophage Induces Malignancy Progression}

Haiyan Piao

Liaoning Cancer Institute and Hospital

Lingfeng Fu

Kumamoto University Faculty of Life Sciences School of Medicine: Kumamoto Daigaku Daigakuin

Seimei Kagaku Kenkyubu Igakubu

Yang Liu

China Medical University

Yue Wang

Liaoning Cancer Institute and Hospital

Xiangyu Meng

Liaoning Cancer Institute and Hospital

Dong Yang

Liaoning Cancer Institute and Hospital

Xiang Xiao

shanghai yanji biomedical technology

Jun Zhang ( $\square$ surgeonzhangjun@hotmail.com )

Kumamoto University Faculty of Life Sciences School of Medicine: Kumamoto Daigaku Daigakuin

Seimei Kagaku Kenkyubu Igakubu https://orcid.org/0000-0003-1739-6462

\section{Research Article}

Keywords: Gastric cancer, macrophage, positive feedback loop, hypoxia, cytokines, signaling pathway

Posted Date: January 7th, 2022

DOI: https://doi.org/10.21203/rs.3.rs-1186986/v1

License: (c) (1) This work is licensed under a Creative Commons Attribution 4.0 International License.

Read Full License 


\section{Abstract}

Background: Hypoxia and inflammation tumor microenvironment (TME) play a crucial role in tumor development and progression. Although increased understanding of TME contributed to gastric cancer (GC) progression and prognosis, the direct interaction between macrophage and GC cells was not fully understood.

Methods: Hypoxia and normoxia macrophage microarrays of GEO database was analyzed. The peripheral blood mononuclear cell acquired from the healthy volunteers.

The expression of CXCL8 in GC tissues and cell lines was detected by quantitative reverse transcription PCR (qRT-PCR), western-blot, Elisa and immunofluorescence. Cell proliferation, migration, and invasion were evaluated by cell counting kit 8 (CCK8), colony formation, real-time imaging of cell migration and transwell. Luciferase reporter assays and chromatin immunoprecipitation were used to identify the interaction between transcription factor and target gene. Especially, a series of truncated and mutation reporter genes were applied to identify precise binding sites.The corresponding functions were verified in the complementation test and in vivo animal experiment.

Results: Our results revealed that Hypoxia triggered macrophage secreted C-X-C Motif Chemokine Ligand 8 (CXCL8), which induced GC invasion and proliferation. This macrophage-induced GC progression was CXCL8 activated C-X-C Motif Chemokine Receptor 1/2 (CXCR1/2) on the GC cell membrane subsequently hyperactivated Janus kinase 1/ Signal transducer and activator of transcription 1 (JAK/STAT1) signaling pathway. Then, the transcription factor STAT1 directly led to the overexpression and secretion of Interleukin 10 (IL-10). Correspondingly, IL-10 induced the M2-type polarization of macrophages through the Nuclear Factor kappa B (NF-кB) pathway-dependent mechanism and continued to increase the expression and secretion of CXCL8 through the transcription factor Nuclear Factor Kappa B Subunit 1 (NFKB1, p50). It suggested a positive feedback loop between macrophage and GC. In clinical GC samples, increased CXCL8 predicted a patient's pessimistic outcome.

Conclusion: Our work identified a positive feedback loop governing cancer cells and macrophage in GC that contributed to tumor progression and patient outcome.

\section{Background}

Gastric cancer (GC) is a prevalent yet incurable malignancy of the digestive system. It is the 3rd leading cause of cancer-related mortalities across the globe ${ }^{1}$. China has a high GC incidence and a heavy disease burden, with an estimated 320,000 annual deaths, accounting for $45 \%$ of all GC deaths globally ${ }^{2}$. Despite the constant iterations of comprehensive therapy based on surgical resection, GC prognosis remains poor, with a 5 -year survival rate of not more than $30 \%{ }^{3}$. On one hand, this is attributed to the malignant phenotype of the tumor; while on the other hand, the tumor microenvironment plays an essential role as an "accomplice" 4 . Hypoxia and inflammation are essential components of the tumor 
microenvironment (TME) ${ }^{5}{ }^{6}$. Previous studies confirmed that hypoxia promotes GC progression ${ }^{7}$. Moreover, the hypoxia-related core element, hypoxia-inducible factor-1 a (HIF-1a) were confirmed as transcription factors that could broadly regulate the transcription of downstream genes, promoting GC proliferation ${ }^{8}$ and invasion ${ }^{9} 10$. Therefore, it is essential to further explore the crosstalk between hypoxia and inflammation in the GC-TME.

Macrophages are the most abundant inflammatory cells in the tumor microenvironment. They exhibit significant plasticity and can freely switch from one phenotype to another; however, this depends on the signals received from their surrounding microenvironment ${ }^{11}{ }^{12}$. The process is called macrophage polarization. Based on the activation state, macrophages can be classified into classically activated macrophages (M1 macrophages) and alternatively activated macrophages (M2 macrophages) ${ }^{13}$. Although this dichotomy is somewhat arbitrary, it remains the most popular macrophage definition ${ }^{14} 15$. It is generally believed that the M1 type has an anti-tumor property, whereas the M2 type is a pro-cancer factor. Also, the cancer cell is an "educator" that converts M1 to M2 ${ }^{16} . \mathrm{M} 2$ macrophages promote tumor metastasis and angiogenesis by secreting various cytokines and exerting immunosuppressive effects ${ }^{17}$. Macrophages recruited into TME by cytokines are referred to as tumor-associated macrophages (TAMs) 18. In TME, TAMs predominantly exhibit an M2 phenotype, indicating a poor prognosis of solid tumors ${ }^{19}$ ${ }^{20}$. Since hypoxia could promote immune evasion, angiogenesis, proliferation, and metastasis, research suggests that it can as well facilitate TAMs in tumor development. Additionally, the TAMs in tumor hypoxic regions mediate treatment resistance and promote cancer recurrence ${ }^{21}$.

This paper focuses on the effect of hypoxia on macrophages then analyzes the role of hypoxic microenvironment and inflammatory microenvironment in GC development. CXCL8 (C-X-C Motif Chemokine Ligand 8 , also called $I L-8$ ) was overexpressed in hypoxic macrophages. Moreover, we discovered that the macrophage-derived CXCL8 could activate the JAK/STAT1 signaling pathway and promote GC invasion as well as proliferation. Besides, as a transcription factor, STAT1 could upregulate IL-10 expression (Interleukin 10) in GC; the latter could naturally trigger macrophage M2-type polarization. The M2 macrophages increased the release of $C X C L 8$. The hypoxia-activated positive feedback loop CXCL8/CXCR1/2 ( C-X-C Motif Chemokine Receptor 1/2)/STAT1/IL-10/NFKB1/CXCL8 could cascade and amplify the interaction between $\mathrm{GC}$ and macrophages leading to uncontrolled progressive signaling in GC.

\section{Materials And Methods}

\subsection{Bioinformatics}

Hypoxic and normoxic cultured human monocyte-derived macrophage dataset GSE4630 was downloaded from the GEO (Gene Expression Omnibus) database (https://www.ncbi.nlm.nih.gov/geo/). They included two hypoxic-cultured samples and two normoxic-cultured samples. The GC RNA sequencing data were downloaded from the TCGA (The Cancer Genome Atlas, TCGA-STAD, 375 tumor 
samples vs. 31 normal samples, https://portal.gdc.cancer.gov/). The GC and normal sample microarray data were also obtained from the GEO (GSE54129, 111 tumor samples vs. 21 normal samples). The differentially expressed genes were identified by the "limma" package $(P<0.05$, and |FoldChange| $\geq 2)$. JASPAR (http://jaspar.genereg.net/) and UCSC (http://genome.ucsc.edu/) were performed to predict the transcription factor.

\subsection{Cell culture and human PBMCs isolation}

The GC (HGC-27, NUGC3) cells, human normal gastric epithelial cell line (GES-1) were acquired from China Medical University (Shenyang, China). Peripheral blood mononuclear cells (PBMCs) were obtained from healthy donors in Liaoning Cancer Hospital \& Institute. CD14 ${ }^{+}$cells were enriched by depleting CD8 ${ }^{+}$, CD19 ${ }^{+}, \mathrm{CD} 56+$ and CD14+ cells by MACS (magnetic-activated cell sorting, EasySep, STEMCELL Technologies, 19059) columns with negative selection. PBMCs were differentiated into M0 macrophages using $50 \mathrm{ng} / \mathrm{mL}$ human macrophage colony-stimulating factor (hM-CSF, Sigma, 81627-83-0) for 7 days (Fig. 1C). All cells were cultured in RPMI-1640 (FUJIFILM Wako Pure Chemical Corporation, 189-02145) supplemented with $10 \%$ fetal bovine serum (FBS, Gibco $26140-079$ ) under $1 \% \mathrm{O}_{2}$ or $20 \% \mathrm{O}_{2}$ conditions as described previously 81022 .

\subsection{GC tissues and ethical approval}

Between January 2009 and December 2012, gastric cancer and adjacent non-cancerous tissue samples were obtained from 526 patients subjected to adequate complete surgical resection (R0) of locally advanced GC surgical resection in Liaoning Cancer Hospital \& Institute. All recruited patients had not received preoperative chemotherapy or radiotherapy. They signed a written informed consent before surgery. The follow-up of patients was closed on December 31,2020. The study was approved by the Ethics Committee of Liaoning Cancer Hospital and Research Institute (20181226).

\subsection{Immunohistochemistry}

The immunohistochemistry (IHC) was performed based on previously published protocols ${ }^{23}$. Immunohistochemistry was scored based on the intensity of staining and the proportion of positive cells. The blinded review was performed by two pathologists. Ki67 (1:1000, Abcam, ab15580), Caspase-3 (1:1000, Abcam, ab184787) CXCL8 (5 $\mu \mathrm{g} / \mathrm{mL}$, R\&D Systems, AF-208-NA).

\subsection{Immunofluorescence}

The slices were overnight incubated with primary antibody at $4^{\circ} \mathrm{C}$. Then, they were incubated with species-appropriate rabbit/mouse secondary antibodies coupled with AlexaFluor dyes $(488,594,1: 200$, Invitrogen, A32814, A32723, A32740), DAPI (1:1000, Dojindo, KT013) at room temperature for 1 hour.

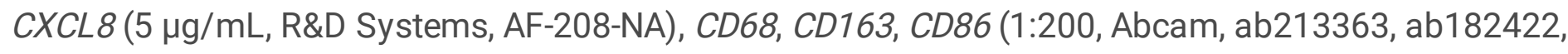
ab270719). Olympus Fluoview FL1200 confocal microscope was used to capture 20x and 60x images. 


\subsection{ELISA test}

ELISA assay was used to measure the CXCL8 in the supernatant of macrophages cultured in hypoxic or normoxic conditions and $I L-10$ in the medium of GC cells. The procedure followed the instructions of the Human CXCL8 kit (R\&D Systems, D8000C) and Human IL-10 ELISA Kit (Abcam, ab185986).

\section{7 qRT-PCR}

The quantitative real-time polymerase chain reaction (qRT-PCR) was performed as previously documented ${ }^{8}$. Primer sequences were designed by Sangon (China, Supplementary Table 1).

\subsection{Western blot analysis}

The RIPA lysis buffer was used to extract total proteins from cells and tissues (Beyotime, Shanghai, China). Then, $10 \%$ SDS-PAGE gel was used to separate the proteins and transferred using the PVDF membrane. The membranes were overnight incubated with the primary antibodies (Supplementary Table 2) at $4^{\circ} \mathrm{C}$. Subsequently, they were co-cultured with the secondary antibody for $1 \mathrm{~h}$. The ECL system (Amersham Imager 600) and Image J software were applied to observe and calculate grayscale values ${ }^{8}$.

\subsection{Chromatin immunoprecipitation (ChIP)}

ChIP enzymatic chromatin IP kit protocol (Cell Signaling Technology, China) was used to perform the ChIP assay, where $1 \times 10^{7}$ logarithmic phase cells were subjected to ChIP. The chromatin was immunoprecipitated with an anti-STAT1 (Santa Cruz Biotechnology, USA, 1:50) and mouse IgG (Santa Cruz Biotechnology, USA, 1:100) on rotators at $4{ }^{\circ} \mathrm{C}$ for $16 \mathrm{~h}$.

\subsection{Promoter-Luciferase reporter assay}

Promoter-Luciferase reporter assay confirmed the combination of STAT1 and IL-10 promoter sequence, $N F K B 1$, and $C X C L 8$ promoter sequence, respectively. The recombinant $p G L-3$ basic-plasmid contained truncated human $I L-10$ (or CXCL8) promoter (wild type, wt, -2000 +99) or mutant IL-10 (or CXCL8) promoter (mutant type, mut). The plasmids were transfected with the POLO3000 transfection reagent (Research and science, China). After $48 \mathrm{~h}$, the luciferase activity of each group was evaluated following the manufacturer's instructions.

\subsection{Colony formation assay}

A total of $1 \times 10^{5} \mathrm{GC}$ cells/each group were seeded into 6-well plates for 2 weeks. The colonies were washed three times using PBS, fixed with $4 \%$ paraformaldehyde, and stained with Diff-Quik III Kit (Muto Chemical, ZS0003). The stained colonies were observed under a light microscope (IX81 Olympus).

\subsection{Cell counting assay}


Cell proliferation was evaluated using the Cell counting kit (Cell counting kit 8, CCK-8). The cells were incubated at $37^{\circ} \mathrm{C}$ for $24,48,72$, and $96 \mathrm{~h}$. Subsequently, the medium was discarded then the chromogenic solution at 10:1 was prepared. For incubation, a color-developing solution (10 $\mu$ l) was added to the 96 well plates at $37^{\circ} \mathrm{C}$ for $2 \mathrm{~h}$. The optical density (OD) was detected using the UV spectrophotometer (BioTek Synergy H1) at $450 \mathrm{~nm}$.

\subsection{Transwell}

Gastric cancer cells were seeded in Transwell upper chambers coated with gelatin. The lower chambers with $600 \mu \mathrm{L}$ RPMI- 1640 with $20 \%$ FBS. After $24 \mathrm{~h}$, the cells were fixed and stained using methanol, hematoxylin, and eosin (Sigma-Aldrich, St. Louis, MO, USA). The upper chamber was removed and the lower layer migrating cells counted under the microscope (IX81 Olympus).

\subsection{Real-time imaging of cell migration}

A total of $200 \mu \mathrm{l}$ Matrigel (BD Biosciences, 356235) pre-coated the bottom of the six-well plates, the cells were then inoculated for $24 \mathrm{~h}$. Thereafter, a 6-well plate was cultured and imaged using KEYENCE BZX700 (KEYENCE, Japan), equipped with $\mathrm{CO}_{2}$ and temperature control chamber as well as a time-lapse tracking system. BZ-X Viewer software (KEYENCE) was used to capture phase-contrast images at intervals of every 10 minutes for 48 hours, while the BZ-X Analyzer software (KEYENCE) was used to convert the continuous images into movie files. Furthermore, KEYENCE video editing and analysis software was used to analyze cell migration in the movies. Microsoft Excel 2010 was used to process the trace data to create $\mathrm{XY}$ coordinate graphs and distance measurements.

\subsection{Xenograft mouse model}

For subcutaneous tumor xenografts, an estimated $1 \times 10^{6} \mathrm{GC}$ cells $/ 0.2 \mathrm{ml}$ PBS (with or without Recombinant Human CXCL8) were subcutaneously injected into the right axillary region of a 5-week-old female BALB/ C mouse. After 28 days of injection, tumors were collected, measured volumes weighed, and photographed. The volume was calculated using the following formula (tumor volume $=L * W * W / 2$ ).

For the intraperitoneally model, approximately $5 \times 10^{6} \mathrm{GC}$ cells $/ 0.1 \mathrm{ml}$ PBS (with or without Recombinant Human CXCL8) were injected into the mouse as mentioned above. Similarly, the mice were euthanized 28 days after injection, then the volume of ascites and the number of visible $(>0.1 \mathrm{~cm})$ metastatic nodules in the peritoneal cavity were measured.

\subsection{Other reagent or resource}

Recombinant Human IL-8 (CXCL8, PeproTech, 200-08), Recombinant Human IL-10 (PeproTech, 200-10), Recombinant Human M-CSF (PeproTech, 300-25), Repertaxin (Sigma-Aldrich, 266359), Fludarabine (Tocris, 3495/10), Sarsasapogenin (Selleck, S3607).

\subsection{Statistical analysis}


GraphPad Prism 9.0 (GraphPad Software Inc) and SPSS 24.0 statistical software (IBM) were used for statistical analyses of data. The student's $t$-test was utilized to perform statistical analysis. $P<0.05$ was considered statistically significant.

\section{Results}

\subsection{Hypoxia promotes macrophage-derived CXCL8 secretion}

Relevant expression profile data were downloaded from the GEO database (GSE4630) to identify the reflection of macrophages under hypoxic conditions. The dataset included four PBMCs-derived macrophages samples. Two of them were cultured in $0 \% \mathrm{O}_{2}$, while the other two were cultured in $20 \% \mathrm{O}_{2}$. The "limma" package was used to identify the differentially expressed genes (DEGs, Supplementary Table 3). As a type of immune cell, macrophages could disrupt the microenvironment through cytokines. Thus, this work assessed the effect of hypoxia on cytokine-related gene expression. Among them, CXCL8 was significantly upregulated in the hypoxic-cultured macrophage (Fig. 1A, 1B). This implies that hypoxic TME might stimulate CXCL8 secretion by macrophages; nevertheless, this warrants further validation. CD14+ positive monocytes by MACS (magnetic-activated cell sorting) negative selection were obtained from healthy volunteers to accurately reflect the in vivo features of macrophages. The PBMCs were induced into M0 macrophage (M0) for 7 days (Fig. 1C). Subsequently, incubation of M0 for $72 \mathrm{~h}$ was continued under hypoxic $\left(1 \% \mathrm{O}_{2}\right)$ and normoxic $\left(20 \% \mathrm{O}_{2}\right)$ conditions, respectively. Both qRT-PCR and Western-blot showed that $C X C L 8$ was overexpressed in hypoxic-cultured MO (Fig. 1D, 1E). Similarly, the upregulated CXCL8 in the supernatant of hypoxic-culture MO was confirmed through the ELISA test (Fig. 1F). Immunofluorescence analysis in human GC tissues showed extensive co-localization of CD68 and CXCL8, suggesting that macrophages could secrete CXCL8 (Fig. 1G).

\subsection{CXCL8 correlates with poor prognosis and tumor progression of GC}

CXCL8 expression in TCGA and GEO (GSE54129) was assessed to further elucidate its role in GC. CXCL8 expression was significantly upregulated in GC tissues compared to that in normal tissues (Fig. 2A, 2B). Subsequently, we assessed the clinical significance of $C X C L 8$ based on the Liaoning cancer hospital cohort. CXCL 8 in cancerous tissues was remarkably higher than that in paired paracancerous tissues (Fig. 2C). Figure 2D reveals the representative images of high expression and low expression CXCL 8 in GC tissue. Moreover, high CXCL8 expression promoted poor DFS (disease-free survival, Fig. 2E) and OS (overall survival, Fig. 2F). The cohort information is listed in Exhibit Supplementary Table 4.

\subsection{CXCL8 activates the CXCL8-CXCR1/2 axis of GC and exacerbates the malignant phenotype}

In vitro and in vivo assays were performed to assess the function of CXCL8 on GC cells. The GC cells were seeded on the six-well plates coated with Matrigel. The real-time imaging recorded the motion state of the cells. Migration of GC cells was significantly improved when co-culture with recombinant human CXCL8. CXCL8 accelerated cells migration (50ng/ml, Fig. 3A-3C, Sup movie 1, 2). Nonetheless, the enhanced motility was inhibited by adding CXCL8-CXCR1/2 inhibitors (Repertaxin, $50 \mu \mathrm{g} / \mathrm{ml}$, Fig. 3A-3C, 
Sup movie 1,2). The promoting impact of $C X C L 8$ on GC invasion ability was achieved by the CXCL8CXCR1/2 axis. In keeping with this result, the Transwell assay also exhibiting CXCL 8 caused an increase in the number of cells penetrating the membrane, but was blocked by repertaxin (Fig. 3D). On cell proliferation, the CCK-8 and colony formation assay demonstrated the promotion of cell proliferation by CXCL8 (Fig. 3E, 3F); this was also achieved through the CXCL8-CXCR1/2 axis (Fig. 3E, 3F). In the in vivo assays, we also confirmed that $C X C L 8$ promoted the formation of the subcutaneous tumor. After injection of GC cells stimulated by CXCL8, the subcutaneous tumors had a larger size and heavier weight than the control. In contrast, no significant difference was noted in tumor size between the control and the inhibitor-added groups (Fig. 3G).

Furthermore, unlike other digestive system cancers metastasizing primarily through the vasculature, GC cells had a greater tendency to develop peritoneal metastases. Therefore, we evaluated the effect of CXCL8 on these types of metastases. After intraperitoneal GC cells co-culturing with $C X C L 8$, the mice evolved peritoneal metastases, but it did not appear in both control and inhibitor groups (Fig. 3H). This implies that $C X C L 8$ could promote GC malignant phenotype; this was achieved through the CXCL8CXCR1/2 axis.

\subsection{JAK/STAT1 pathway is downstream of CXCL8}

After clarifying the oncogenic effect of $C X C L 8$ on GC, it is essential to explore the possible mechanisms involved. Since JAK/STAT1 pathway is a canonical downstream of the Chemokine signaling pathway 24 25 , the effect of the CXCL8-CXCR1/2 axis on the JAK/STAT1 pathway activation was assessed. The protein expression of $p-J A K, p-S T A T 1$ was significantly upregulated after stimulation of GC with $C X C L 8$, while the expression of JAK, STAT1 did not significantly change (Fig. 4A). Similarly, the CXCL8-CXCR1/2 inhibitors and repertaxin inhibited the activation of the JAK/STAT1 pathway by CXCL8 (Fig. 4A). JAK/STAT1 pathway might be the downstream effector of the CXCL8-CXCR1/2 axis. To validate the result, CXCL8-induced GC cells were co-cultured with STAT1 specific inhibitor (Fludarabine). As expected, the activation effect of $C X C L 8$ on JAK/STAT1 disappeared when cells were treated with Fludarabine $(50 \mu \mathrm{M}$, Fig. 4A). This confirmed that the JAK/STAT1 is the downstream effector of CXCL8. Unsurprisingly, the JAK/STAT1 pathway inhibitors also limited the effects of $C X C L 8$ on invasion (Fig. 4B-4E, Sup movie 3,4 ) and proliferation (Fig. 4F, 4G). Thus, a preliminary conclusion would be exogenous CXCL8 continuity activated the CXCL8/CXCR1/2-JAK/STAT1 pathway and triggered invasion and proliferation of GC.

\subsection{STAT1 regulates the expression of IL-10 directly but not HIF-1a}

STAT1 belongs to the STAT protein family that forms homo- or hetero-dimers and translocates to the nucleus to exert regulatory effects as a transcriptional activator. Naturally, we sought to identify the downstream regulatory molecules of STAT1. Through the JAPAR database, the target genes of STAT1 were predicted. Among them, HIF-1 $a$ and IL-10 attracted our attention. The former was the hypoxic associated core effector molecule, while the latter was closely related to the polarization of macrophages. It should assess the influence of STAT1 on HIF-1a and IL-10. The shRNA and OE-vector of STAT1 could 
up-or down-regulated STAT1 expression (Fig. 5A). STAT1 inhibition was followed by a downregulation in HIF-1 $a$ and $I L-10$ expressions both at the transcriptional and protein levels (Fig. 5B-5E). In contrast, both were upregulated with STAT1 overexpression (Fig. 5B-5E). With the promising results, luciferase reporter assay and ChIP were performed to assess the interaction of STAT1 with HIF-1 $a$ and IL-10 promoters. Therefore, full-length HIF-1 $a$ and $I L-10$ promoters were cloned into luciferase reporter plasmids, respectively. The luciferase reporter assay revealed that the up-regulated STAT1 could enhance the luciferase activity of $I L-10-w t$, but not $I L-10-m u t$ (Fig. 5F). Unfortunately, STAT1 did not affect the fluorescence intensity of HIF-1a, whether HIF-1a-wt or HIF-1a-mut (Fig. 5G). This suggests that as a transcription factor, STAT1 could directly activate the transcription of IL-10, however, HIF-1a promotion might rely on other indirect pathways. Subsequently, the ChIP assay confirmed that the STAT1 antibody could dismantle the $I L-10$ sequence but not the control IgG (Fig. $5 \mathrm{H})$. A series of reporter genes comprising truncated $I L-10$ promoter sequences were constructed to further refine the target sequence of the promoter. The luciferase reporter assay showed that deleting the region between -1811 and -1821 bases of $I L-10$ promoter severely abolished IL-10 by STAT 1 activation (Fig. 5I).

\subsection{IL-10 induces M2 polarization through the NF-KB pathway and promotes CXCL8 expression}

As evidenced above, hypoxic-macrophage-derived $C X C L-8$ could promote $I L-10$ expression by activating JAK/STTA1 in GC while the cytokine $I L-10$ can induce M2 polarization. Therefore, the effect of $I L-10$ on macrophages and the potential underlying mechanisms should be confirmed. Unsurprisingly, immunofluorescence confirmed that $I L-10$ significantly promoted $C D 163$ expression in PBMCs induced macrophage, and M2 polarization (Fig. 6A). Of note, the inhibition of the NF-KB pathway is an essential factor in driving macrophage $M 1$ polarization ${ }^{26}$. Thus, it is necessary to evaluate the effect of $I L-10$ on the NF-KB pathway in macrophages. The addition of $I L-10$ upregulated $p 50$ and p65 expression in the

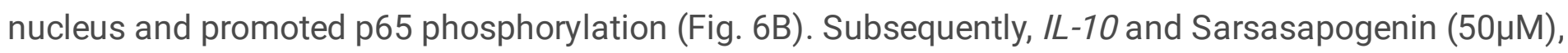
an inhibitor, specifically targeting $I K B$ were used to inhibit the $N F-K B$ pathway and co-cultured macrophages. Sarsasapogenin attenuated $C D 163$ expression (Fig. 6C). It inhibited the M2 polarization. This demonstrates that the effect of $I L-10$ on macrophage M2 polarization was partly achieved through the NF-KB pathway.

Besides, we analyzed the effects of $I L-10$ and NF-KB pathways on CXCL8 expression. IL-10 upregulated CXCL8 expression at the transcriptional and protein levels (Fig. 6D, 6E) and intensified the secretion of the latter (Fig. 6F). Correspondingly, the NF-KB pathway could antagonize this effect (Fig. 6D-6F). This implies that the IL-10-NF-KB axis was implicated in CXCL8 regulation at the transcriptional level. The subsequent transcription factor luciferase reporter assay and ChIP confirmed our suspicions. The gene (p50) was bound to the promoter of CXCL8 (Fig. 6G, 6H). A series of truncated CXCL8 promoter sequences were constructed, which included at least one potential binding site (in red). Nevertheless, every sequence could promote $C X C L 8$ expression. Mutated the mut1 (in blue, -1829 -1819) and mut2 (in green, $-91 \sim-81$ ) sequences simultaneously, the fluorescence intensity significantly decreased. This confirmed the presence of two NFKB1 binding sites in the promoter sequence and up-regulated CXCL8 expression. 
In this way, a positive feedback loop was formed between macrophage and GC cells that exacerbated tumor deterioration under the catalysis of hypoxic TME.

\section{Discussion}

TME of most solid tumors is characterized by hypoxia and inflammation ${ }^{27}$. The crosstalk between TME and cancer cells promotes tumor growth and progression ${ }^{28}$. Cytokines are the critical bridge that maintains this intercellular communication; besides, they can favorably shape the TME for tumor cells ${ }^{29}$. Gastric cancer cells rely on this complex environment to achieve sustainable development, invasion, and metastasis. In our previous study, we continuously focused on the effect of hypoxia on the progression of GC cells ${ }^{30}$. Nevertheless, this effect on TME stromal cells and inflammatory cells has been overlooked.

Of note, tumor-infiltrating macrophages release the strongest signal in inflammatory TME ${ }^{31}$. Thus, we concentrated on the effect of hypoxia on macrophage cytokines i.e., the communication tools. Among them, CXCL8 exhibited the most potent response to the hypoxic signal. CXCL8 is one of the earliest and most comprehensively studied chemokines. It was discovered, purified, and sequenced in monocytes in the 1980 s $^{32}$. CXCL 8 is secreted by different cells, including monocytes, alveolar macrophages, fibroblasts, endothelial cells, and epithelial cells ${ }^{33} 34$. Additionally, cytokines (interleukin-1, interleukin-6, CXCL12, TNFa), hypoxia, reactive oxygen species (ROS), bacterial particles could stimulate CXCL8 expression. The transcription factor $N F-K B$ and activator protein-1 ( $A P-1)$ were also implicated in $C X C L 8$ activation ${ }^{35} 36$. Notably, CXCL8 was nearly undetectable in unstimulated cells. In contrast, the above stimulation would upregulate $C X C L 8$ expression by 10 to 100 times $^{37}$. Besides, this type of explosive growth provides a basis for its role in cancers. CXCL8 binds $\mathrm{G}$ protein-coupled receptors or CXCR $1 / 2$ to transmit its signal. $C X C L 8-C X C R 1 / 2$ axis stimulates endothelial cells and promotes tumor angiogenesis 38. Blocked CXCL8-CXCR2 signal transmission between TAMs and cancer cells could improve the effect of anti-PD1 treatment ${ }^{39} 40$. This study found that the CXCL/8-CXCR1/2 axis activated the JAK/STAT1 pathway in GC, thereby promoting the malignant phenotype. Further, STAT1 acted as a transcription factor directly regulating $I L-10$ expression, this was closely associated with macrophages. In addition, it could ignore that STAT1 also affected the HIF-1a expression, which was the most crucial element for the organism responding to hypoxia. The indirect regulatory pattern of hypoxia/CXCL8/CXCR1/2/JAK/STAT1/HIF-1a might be a non-classical adaptation pattern of GC cells to the hypoxic microenvironment.

$I L-10$ is one of the most famous molecules causing macrophage polarization ${ }^{41}$. It triggers $M 2$ polarization by regulating the NF-KB and JAK/STAT pathway ${ }^{42}$. Generally speaking, NF-KB pathway activation is often regarded as a marker of $\mathrm{M} 1$ macrophages because of its pro-inflammatory effects. However, with the introduction of the subclassification theory of M2 macrophages, the M2d subtype is the closest to TAMs, and the activation of NF-KB pathway is also a sign event of M2d activation 4344 . This means that the activation of the NF-KB pathway in the complex TME is still a double-edged sword. Subsequently, it was natural for us to further evaluate its effect on macrophage and macrophage-derived 
$C X C L 8$. Our results demonstrated that $I L-10$ regulated $M 2$ polarization via the $N F-K B$ pathway and affected $C X C L 8$ expression. Surprisingly, among the macrophage added with $I L-10$ and NF-KB inhibitors, a few macrophages expressed both $\mathrm{M} 1$ and $\mathrm{M} 2$ type markers, presenting a hybrid state. Although the simple dichotomy of macrophage is widely used, researchers continue to express doubts. With the advancement of single-cell technology, further sub-classification of macrophage by markers on the surface of the macrophage membrane deepen the understanding of the TME ${ }^{45}$. Specifically, tissueresident macrophages and monocyte-derived macrophages have entirely different biological functions, however, the two co-exist in TME ${ }^{19}$. Subsequent luciferase reporter assays and ChIP assays confirmed the regulatory role of the transcription factor NFKB1 (p50) on CXCL8. This was also consistent with previous findings 4647 . Thus, macrophage-derived CXCL8 could further promote the deterioration of GC and cause $I L-10$ expression, recurrence of the cycle.

The above results illustrate a novel paradigm of GC-TAMs' interaction: Hypoxia spurs the release of overexpressed $C X C L 8$ by macrophages which then activates the CXCL8/CXCR1/2-JAK/STAT 1 pathway and promotes GC progression. Moreover, this pathway directly promotes the expression of GC-derived IL10 , further accelerating the release of macrophage-derived $C X C L 8$ under the $/ L-10 / N F-K B / C X C L 8$ axis. In this process, the M2-type polarization is simultaneously induced. This causes ordinary macrophages involved in immune surveillance to "betray" into TAMs implicated in immune escape and suppression. Hypoxia/CXCL8/CXCR1/2-JAK/STAT1/IL-10/NF-KB/CXCL8 forms a positive feedback loop between GC and macrophage (or TAMs). As the core molecule of this circuit, $C X C L 8$ is a high-risk factor for GC prognosis. This suggests that the signaling loop is a potential therapeutic target. Also, research on antiCXCL-8 therapy in solid tumors recruits or collates data (NCT01831310, NCT02536469, NCT04347226), and we have reason to expect these corresponding results.

\section{Conclusion}

In conclusion, our work identifies a hypoxic-activated, inflammation-associated molecular network; which involve CXCL8, CXCR1/2, JAK/STAT1, IL-10, and NF-KB signaling pathways that regulate a positive feedback loop between GC cells and TAMs, tumor progression, macrophage polarization, as well as patient outcomes (Fig. 7). These results indicate the potential of this feedback loop as a therapeutic target for GC.

\section{Abbreviations}

GC: Gastric cancer

TME: Tumor microenvironment

HIF-1a: Hypoxia-inducible factor-1a

TAMs: Tumor-associated macrophages 
CXCL8/IL-8: C-X-C Motif Chemokine Ligand 8

IL-10: Interleukin 10

CXCR1/2: C-X-C Motif Chemokine Receptor 1/2

JAK: Janus Kinase 1

STAT1: Signal Transducer And Activator Of Transcription 1

GEO: Gene Expression Omnibus

TCGA: The Cancer Genome Atlas

PBMC: Peripheral blood mononuclear cell

FBS: Fetal bovine serum

IHC: Immunohistochemistry

qRT-PCR: quantitative real-time polymerase chain reaction

ChIP: Chromatin immunoprecipitation

CCK8: Cell counting kit 8

OD: Optical density

DEGs: Differentially expressed genes

MACS: Magnetic-activated cell sorting

DFS: Disease-free survival

OS: Overall survival

\section{Declarations}

\section{Ethics approval and consent to participate}

The study was reviewed and approved by the Faculty of Science Ethics Committee at Liaoning Cancer Hospital \& Institute (Cancer Hospital of China Medical University, 20181226).

\section{Informed consent}

Informed consent was obtained from patients before surgery at the Liaoning Province Cancer Hospital \& Institute. 


\section{Consent for publication}

All authors agree with the content of the manuscript.

\section{Availability of data and materials}

The data are available from the sources listed in the manuscript-the TCGA and GEO data portal. The data used to support the findings of this study are available from the corresponding author upon request

\section{Competing interests}

The authors declare no conflicts of interest.

\section{Funding}

Liaoning S\&T Project (2020-ZLLH-46), CSCO (Y-HR2019-0337).

\section{Author Contributions}

Piao HY and Fu LF performed the majority of experiments and analyzed the data and drafted the manuscript; Zhang $\mathrm{J}$ designed the research; Liu $\mathrm{Y}$ and Yang D conducted the molecular biology assays and assisted in writing the manuscript; Wang $Y$ and Meng XY collected and analyzed the data. Xiao X contributed to data visualization.

\section{Acknowledgements}

None

\section{Informed consent}

Informed consent was obtained from patients before surgery at the Liaoning Province Cancer Hospital \& Institute.

\section{References}

1. Siegel RL, Miller KD, Fuchs HE, et al. Cancer statistics, 2021. CA: a cancer journal for clinicians 2021;71(1):7-33.

2. Yang L, Zheng R, Wang N, et al. Incidence and mortality of stomach cancer in China, 2014. Chin J Cancer Res 2018;30(3):291-98. doi: 10.21147/j.issn.1000-9604.2018.03.01 [published Online First: 2018/07/27]

3. Galletti G, Zhang C, Gjyrezi A, et al. Microtubule Engagement with Taxane Is Altered in TaxaneResistant Gastric Cancer. Clinical cancer research : an official journal of the American Association for Cancer Research 2020;26(14):3771-83. doi: 10.1158/1078-0432.Ccr-19-3018 [published Online First: 2020/04/24] 
4. Zheng ZQ, Chen JT, Zheng MC, et al. Nestin +/CD31 + Cells in the Hypoxic Perivascular Niche Regulate Glioblastoma Chemoresistance by Upregulating JAG1 and DLL4. Neuro-oncology 2020 doi: 10.1093/neuonc/noaa265 [published Online First: 2020/11/30]

5. Massara M, Bonavita O, Savino B, et al. ACKR2 in hematopoietic precursors as a checkpoint of neutrophil release and anti-metastatic activity. Nat Commun 2018;9(1):676. doi: 10.1038/s41467018-03080-8 [published Online First: 2018/02/16]

6. Lee KE, Spata M, Bayne LJ, et al. Hif1a Deletion Reveals Pro-Neoplastic Function of B Cells in Pancreatic Neoplasia. Cancer Discov 2016;6(3):256-69. doi: 10.1158/2159-8290.Cd-15-0822 [published Online First: 2015/12/31]

7. Zhang J, Wu Y, Lin YH, et al. Prognostic value of hypoxia-inducible factor-1 alpha and prolyl 4hydroxylase beta polypeptide overexpression in gastric cancer. World journal of gastroenterology 2018;24(22):2381-91. doi: 10.3748/wjg.v24.i22.2381 [published Online First: 2018/06/16]

8. Piao H-y, Liu Y, Kang Y, et al. Hypoxia associated IncRNA HYPAL promotes proliferation of gastric cancer as ceRNA by sponging miR-431-5p to upregulate CDK14. Gastric Cancer 2021:1-20.

9. Zhang J, Guo S, Wu Y, et al. P4HB, a Novel Hypoxia Target Gene Related to Gastric Cancer Invasion and Metastasis. BioMed research international 2019;2019:9749751. doi: 10.1155/2019/9749751 [published Online First: 2019/08/31]

10. Zhang J, Jin HY, Wu Y, et al. Hypoxia-induced LncRNA PCGEM1 promotes invasion and metastasis of gastric cancer through regulating SNAl1. Clinical \& translational oncology : official publication of the Federation of Spanish Oncology Societies and of the National Cancer Institute of Mexico 2019;21(9):1142-51. doi: 10.1007/s12094-019-02035-9 [published Online First: 2019/01/29]

11. Murray PJ. Macrophage Polarization. Annu Rev Physio/ 2017;79:541-66. doi: 10.1146/annurevphysiol-022516-034339 [published Online First: 2016/11/05]

12. Okabe Y, Medzhitov R. Tissue-specific signals control reversible program of localization and functional polarization of macrophages. Cel/ 2014;157(4):832-44. doi: 10.1016/j.cell.2014.04.016 [published Online First: 2014/05/06]

13. Hallowell RW, Collins SL, Craig JM, et al. mTORC2 signalling regulates M2 macrophage differentiation in response to helminth infection and adaptive thermogenesis. Nat Commun 2017;8:14208. doi: 10.1038/ncomms14208 [published Online First: 2017/01/28]

14. Stein M, Keshav S, Harris N, et al. Interleukin 4 potently enhances murine macrophage mannose receptor activity: a marker of alternative immunologic macrophage activation. The Journal of experimental medicine 1992;176(1):287-92.

15. Mills CD, Kincaid K, Alt JM, et al. M-1/M-2 macrophages and the Th1/Th2 paradigm. The Journal of immunology 2000;164(12):6166-73.

16. Elinav E, Nowarski R, Thaiss CA, et al. Inflammation-induced cancer: crosstalk between tumours, immune cells and microorganisms. Nature reviews Cancer 2013;13(11):759-71. doi: 10.1038/nrc3611 [published Online First: 2013/10/25] 
17. Ramesh A, Brouillard A, Kumar S, et al. Dual inhibition of CSF1R and MAPK pathways using supramolecular nanoparticles enhances macrophage immunotherapy. Biomaterials 2020;227:119559. doi: 10.1016/j.biomaterials.2019.119559 [published Online First: 2019/11/02]

18. Lu SW, Pan HC, Hsu YH, et al. IL-20 antagonist suppresses PD-L1 expression and prolongs survival in pancreatic cancer models. Nat Commun 2020;11(1):4611. doi: 10.1038/s41467-020-18244-8 [published Online First: 2020/09/16]

19. DeNardo DG, Ruffell B. Macrophages as regulators of tumour immunity and immunotherapy. Nat Rev Immuno/ 2019;19(6):369-82. doi: 10.1038/s41577-019-0127-6 [published Online First: 2019/02/06]

20. Cao Q, Yan X, Chen K, et al. Macrophages as a potential tumor-microenvironment target for noninvasive imaging of early response to anticancer therapy. Biomaterials 2018;152:63-76. doi: 10.1016/j.biomaterials.2017.10.036 [published Online First: 2017/11/08]

21. Henze AT, Mazzone M. The impact of hypoxia on tumor-associated macrophages. The Journal of clinical investigation 2016;126(10):3672-79. doi: 10.1172/jci84427 [published Online First: 2016/08/03]

22. Piao HY, Guo S, Wang Y, et al. Exosome-transmitted IncRNA PCGEM1 promotes invasive and metastasis in gastric cancer by maintaining the stability of SNAI1. Clinical \& translational oncology : official publication of the Federation of Spanish Oncology Societies and of the National Cancer Institute of Mexico 2020 doi: 10.1007/s12094-020-02412-9 [published Online First: 2020/06/11]

23. Piao H-y, Guo S, Jin H, et al. LINC00184 involved in the regulatory network of ANGPT2 via ceRNA mediated miR-145 inhibition in gastric cancer. Journal of Cancer 2021;12(8):2336.

24. Li HS, Watowich SS. Innate immune regulation by STAT-mediated transcriptional mechanisms. Immunol Rev 2014;261(1):84-101. doi: 10.1111/imr.12198 [published Online First: 2014/08/16]

25. Mellado M, Rodríguez-Frade JM, Mañes $S$, et al. Chemokine signaling and functional responses: the role of receptor dimerization and TK pathway activation. Annu Rev Immuno/ 2001;19:397-421. doi: 10.1146/annurev.immunol.19.1.397 [published Online First: 2001/03/13]

26. Locati M, Curtale G, Mantovani A. Diversity, Mechanisms, and Significance of Macrophage Plasticity. Annual review of pathology 2020;15:123-47. doi: 10.1146/annurev-pathmechdis-012418-012718 [published Online First: 2019/09/19]

27. Riera-Domingo $C$, Audigé $A$, Granja $S$, et al. Immunity, Hypoxia, and Metabolism-the Ménage à Trois of Cancer: Implications for Immunotherapy. Physiol Rev 2020;100(1):1-102. doi:

10.1152/physrev.00018.2019 [published Online First: 2019/08/16]

28. Iriondo O, Liu Y, Lee G, et al. TAK1 mediates microenvironment-triggered autocrine signals and promotes triple-negative breast cancer lung metastasis. Nat Commun 2018;9(1):1994. doi: 10.1038/s41467-018-04460-w [published Online First: 2018/05/20]

29. Hamarsheh S, Osswald L, Saller BS, et al. Oncogenic Kras(G12D) causes myeloproliferation via NLRP3 inflammasome activation. Nat Commun 2020;11(1):1659. doi: 10.1038/s41467-020-15497-1 [published Online First: 2020/04/05] 
30. Piao HY, Liu Y, Kang Y, et al. Hypoxia associated IncRNA HYPAL promotes proliferation of gastric cancer as ceRNA by sponging miR-431-5p to upregulate CDK14. Gastric Cancer 2021 doi: 10.1007/s10120-021-01213-5 [published Online First: 2021/07/12]

31. Hakimi AA, Voss MH, Kuo F, et al. Transcriptomic Profiling of the Tumor Microenvironment Reveals Distinct Subgroups of Clear Cell Renal Cell Cancer: Data from a Randomized Phase III Trial. Cancer Discov 2019;9(4):510-25. doi: 10.1158/2159-8290.Cd-18-0957 [published Online First: 2019/01/10]

32. Peveri $P$, Walz A, Dewald B, et al. A novel neutrophil-activating factor produced by human mononuclear phagocytes. J Exp Med 1988;167(5):1547-59. doi: 10.1084/jem.167.5.1547 [published Online First: 1988/05/01]

33. Strieter RM, Kunkel SL, Showell HJ, et al. Monokine-induced gene expression of a human endothelial cell-derived neutrophil chemotactic factor. Biochemical and biophysical research communications 1988;156(3):1340-5. doi: 10.1016/s0006-291x(88)80779-4 [published Online First: 1988/11/15]

34. Wanninger $\mathrm{J}$, Neumeier $\mathrm{M}$, Weigert $\mathrm{J}$, et al. Adiponectin-stimulated CXCL8 release in primary human hepatocytes is regulated by ERK1/ERK2, p38 MAPK, NF-kappaB, and STAT3 signaling pathways. Am J Physiol Gastrointest Liver Physio/ 2009;297(3):G611-8. doi: 10.1152/ajpgi.90644.2008 [published Online First: 2009/07/18]

35. Brat DJ, Bellail AC, Van Meir EG. The role of interleukin-8 and its receptors in gliomagenesis and tumoral angiogenesis. Neuro Oncol 2005;7(2):122-33. doi: 10.1215/s1152851704001061 [published Online First: 2005/04/16]

36. Wald O, Shapira OM, Izhar U. CXCR4/CXCL12 axis in non small cell lung cancer (NSCLC) pathologic roles and therapeutic potential. Theranostics 2013;3(1):26-33. doi: 10.7150/thno.4922 [published Online First: 2013/02/06]

37. Podolin PL, Bolognese BJ, Foley JJ, et al. A potent and selective nonpeptide antagonist of CXCR2 inhibits acute and chronic models of arthritis in the rabbit. $J$ Immunol 2002;169(11):6435-44. doi: 10.4049/jimmunol.169.11.6435 [published Online First: 2002/11/22]

38. Keane MP, Belperio JA, Xue YY, et al. Depletion of CXCR2 inhibits tumor growth and angiogenesis in a murine model of lung cancer. J Immuno/ 2004;172(5):2853-60. doi: 10.4049/jimmunol.172.5.2853 [published Online First: 2004/02/24]

39. Zhang M, Huang L, Ding G, et al. Interferon gamma inhibits CXCL8-CXCR2 axis mediated tumorassociated macrophages tumor trafficking and enhances anti-PD1 efficacy in pancreatic cancer. $J$ Immunother Cancer 2020;8(1) doi: 10.1136/jitc-2019-000308 [published Online First: 2020/02/14]

40. Lin $\mathrm{C}, \mathrm{He} \mathrm{H}$, Liu H, et al. Tumour-associated macrophages-derived CXCL8 determines immune evasion through autonomous PD-L1 expression in gastric cancer. Gut 2019;68(10):1764-73. doi: 10.1136/gutjnl-2018-316324 [published Online First: 2019/01/21]

41. Murray PJ, Allen JE, Biswas SK, et al. Macrophage activation and polarization: nomenclature and experimental guidelines. Immunity 2014;41(1):14-20. doi: 10.1016/j.immuni.2014.06.008 [published Online First: 2014/07/19] 
42. Sica A, Bronte V. Altered macrophage differentiation and immune dysfunction in tumor development. The Journal of clinical investigation 2007;117(5):1155-66. doi: 10.1172/jci31422 [published Online First: 2007/05/04]

43. Wang $\mathrm{Q}, \mathrm{Ni} \mathrm{H}$, Lan $\mathrm{L}$, et al. Fra-1 protooncogene regulates IL-6 expression in macrophages and promotes the generation of M2d macrophages. Cell Res 2010;20(6):701-12. doi: 10.1038/cr.2010.52 [published Online First: 2010/04/14]

44. Xu F, Wei Y, Tang Z, et al. Tumor-associated macrophages in lung cancer: Friend or foe? (Review). Mol Med Rep 2020;22(5):4107-15. doi: 10.3892/mmr.2020.11518 [published Online First: 2020/10/02]

45. Casanova-Acebes M, Dalla E, Leader AM, et al. Tissue-resident macrophages provide a protumorigenic niche to early NSCLC cells. Nature 2021;595(7868):578-84. doi: 10.1038/s41586-02103651-8 [published Online First: 2021/06/18]

46. Karakurum M, Shreeniwas $\mathrm{R}$, Chen J, et al. Hypoxic induction of interleukin-8 gene expression in human endothelial cells. The Journal of clinical investigation 1994;93(4):1564-70. doi:

10.1172/jci117135 [published Online First: 1994/04/01]

47. Frey S, Derer A, Messbacher ME, et al. The novel cytokine interleukin-36a is expressed in psoriatic and rheumatoid arthritis synovium. Ann Rheum Dis 2013;72(9):1569-74. doi: 10.1136/annrheumdis2012-202264 [published Online First: 2012/12/27]

\section{Figures}


A

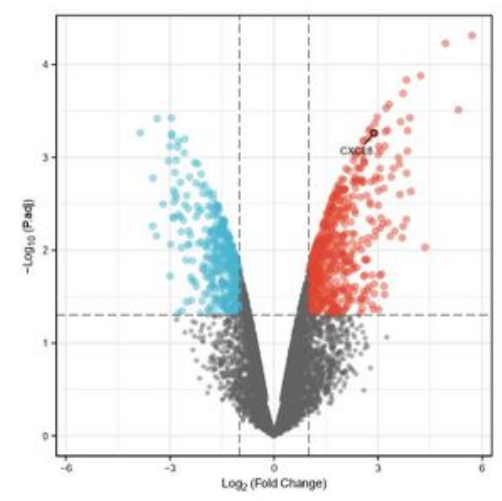

$\mathrm{D}$

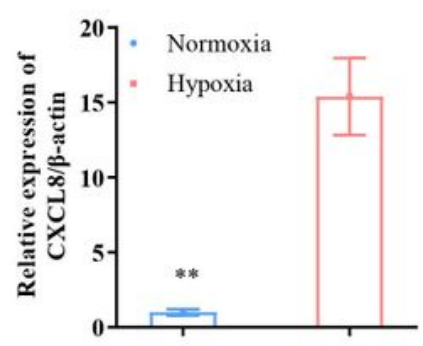

G
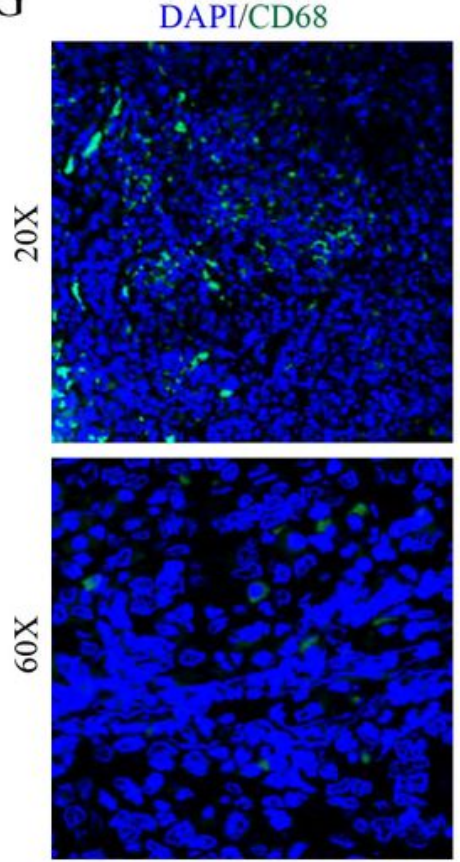

$\mathrm{B}$

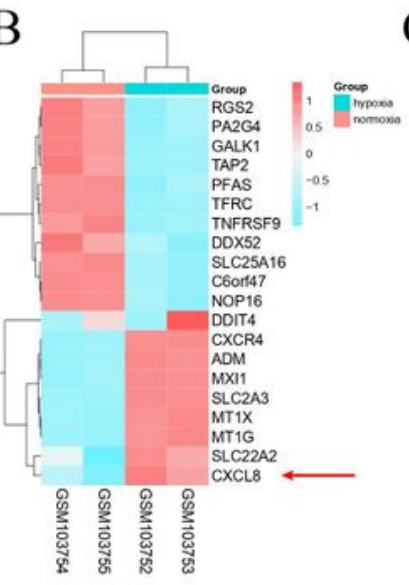

$\mathrm{E}$

$\mathrm{C}$

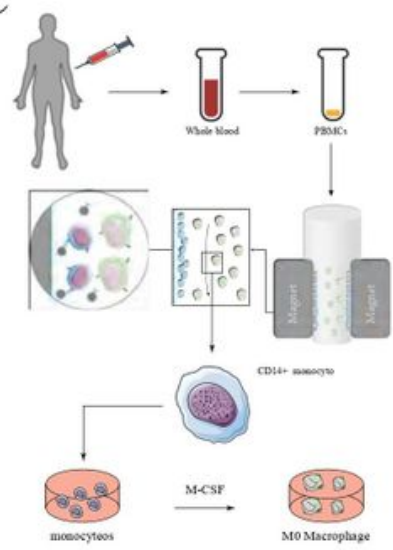

$\mathrm{F}$

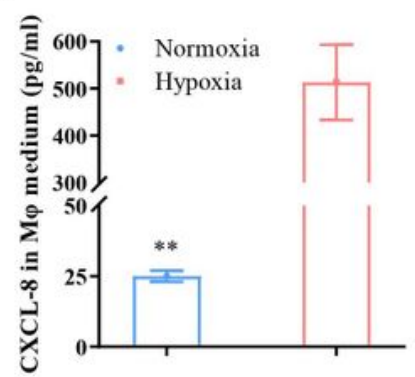

Merge
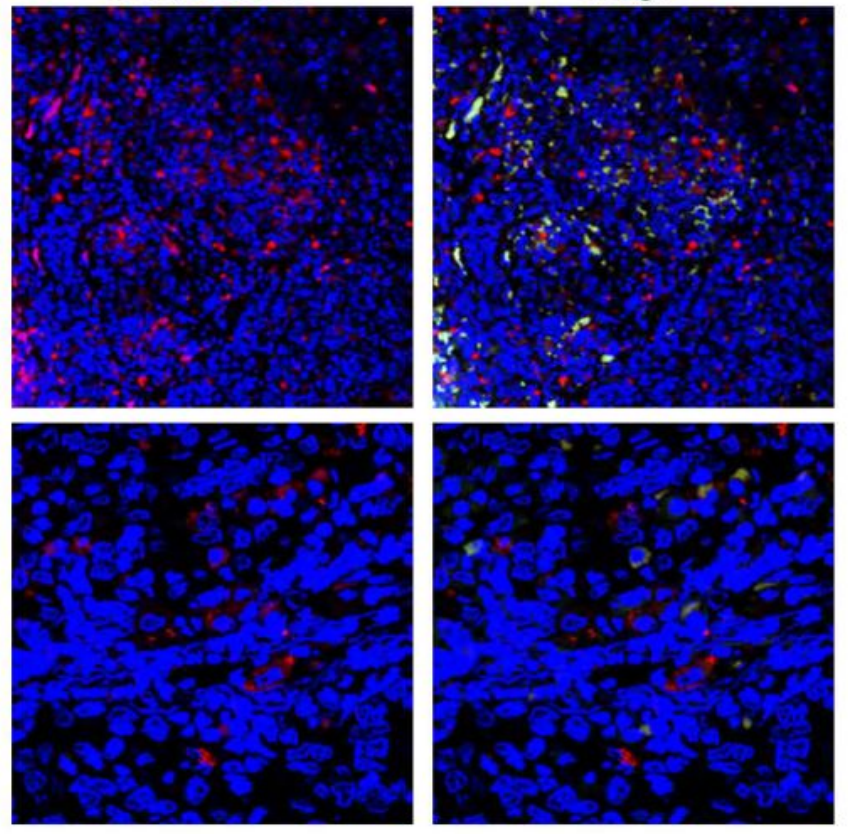

\section{Figure 1}

Hypoxia promoted macrophage-derived CXCL8 secretion

A, B. Differential genetic analysis of the transcription data of macrophages cultured under hypoxia and normoxia showed that hypoxia could promote CXCL8 expression. (A. volcano; B. heatmap) 

C. MACS obtained human $\mathrm{CD} 14^{+}$monocytes and induced them into macrophages through M-CSF.
D, E. Hypoxia promoted human-derived macrophage CXCL8 expression at both transcription and protein levels.

F. Hypoxia promoted the secretion of macrophage-derived cytokine $C X C L 8$ secretion

G. An evident co-localization of macrophages and CXCL8 in human GC tissue specimens.

A
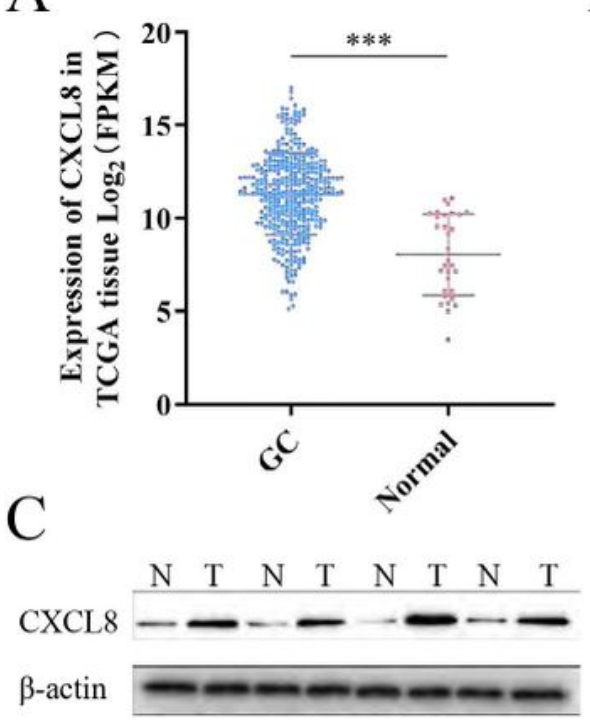

$\mathrm{D}$

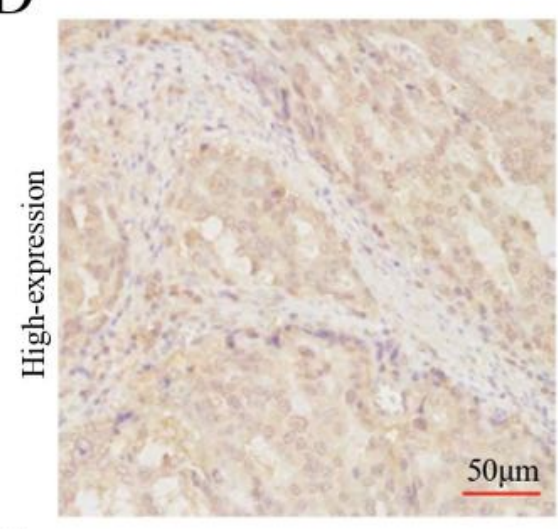

$\mathrm{E}$

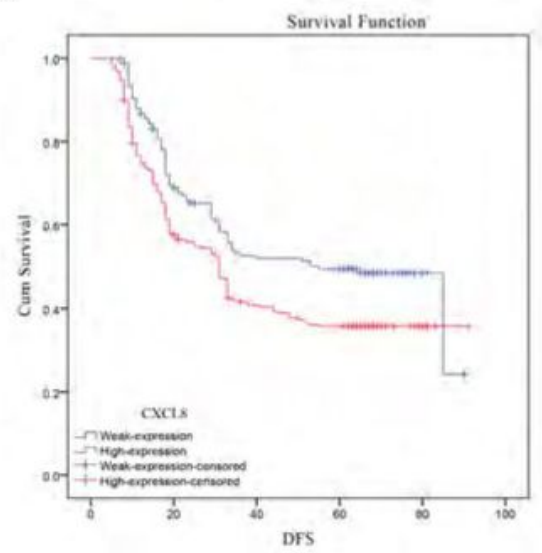

B

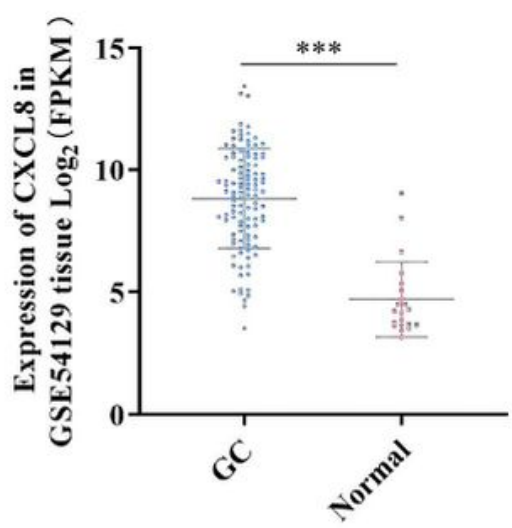

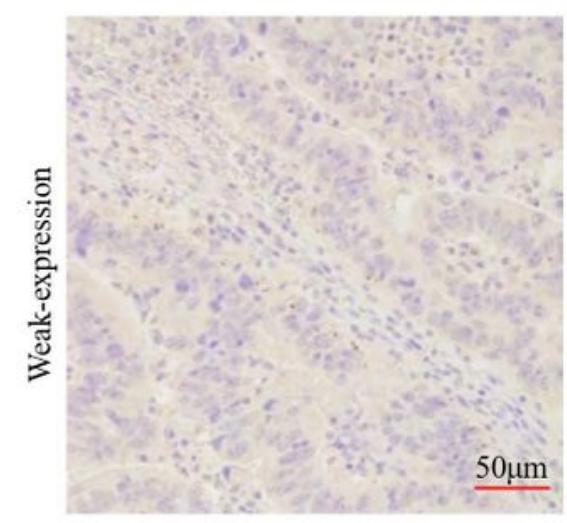

$\mathrm{F}$

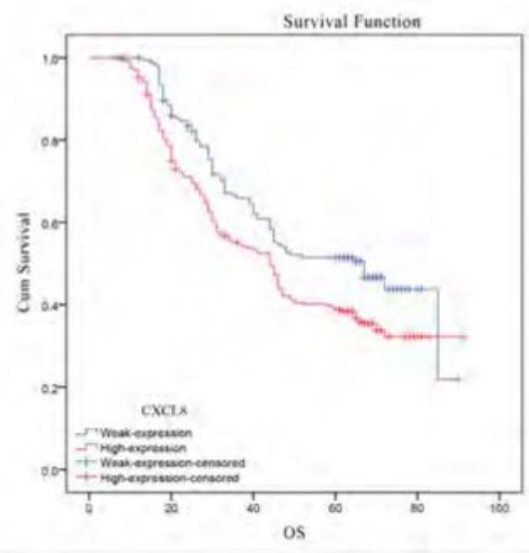


Figure 2

\section{CXCL8 overexpression in GC promotes poor survival.}
A, B. CXCL 8 was up-regulated in GC tissue both in TCGA-GC and GEO-GC cohort.
C. In matched GC and adjacent tissues, CXCL8 was highly expressed in cancer tissues.
D. Representative image of CXCL8 expression in GC tissue. (Left: high-expression; Right: weak- expression).
E, F. CXCL8 promoted poor DFS and OS in the GC cohort. (E. DFS; F. OS.) 
A

A
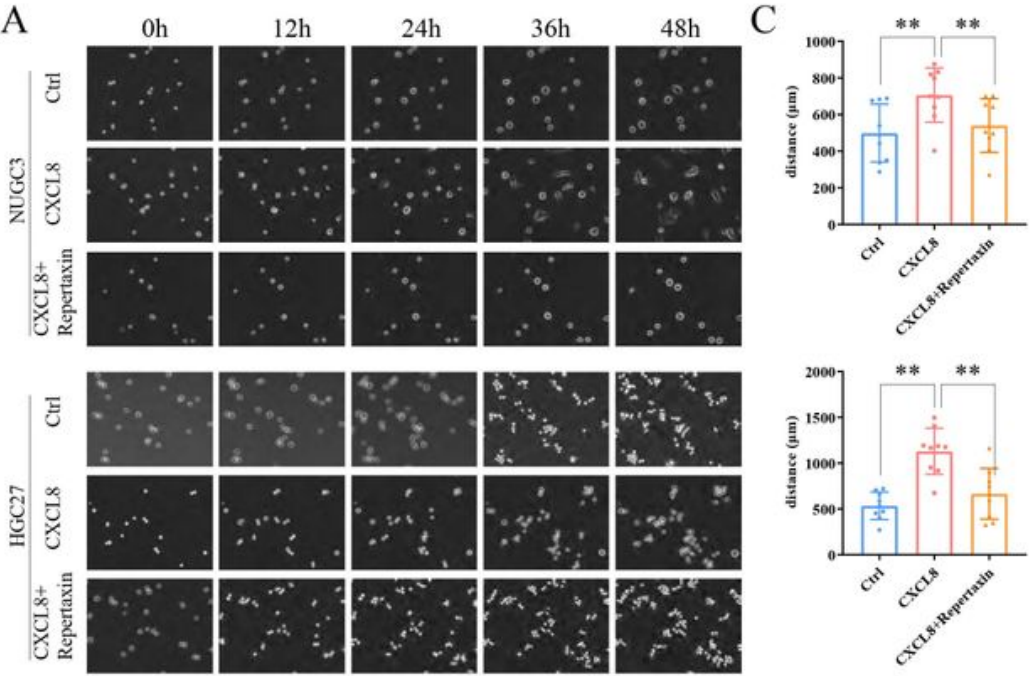

B

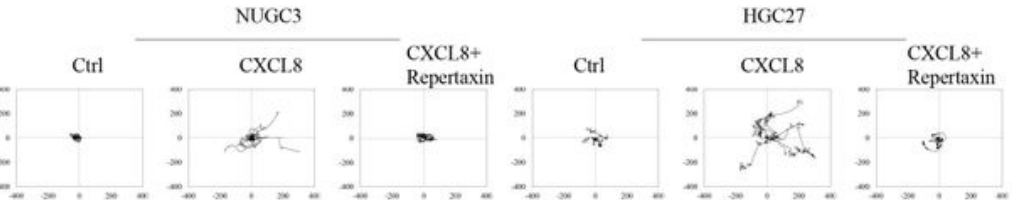

$\mathrm{D}$

$\mathrm{E}$
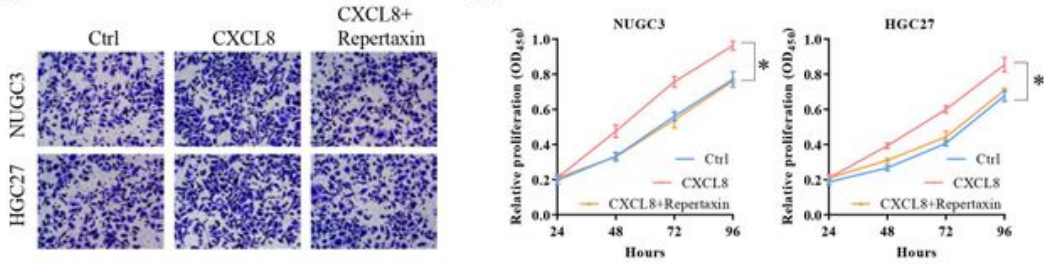

$\mathrm{F}$
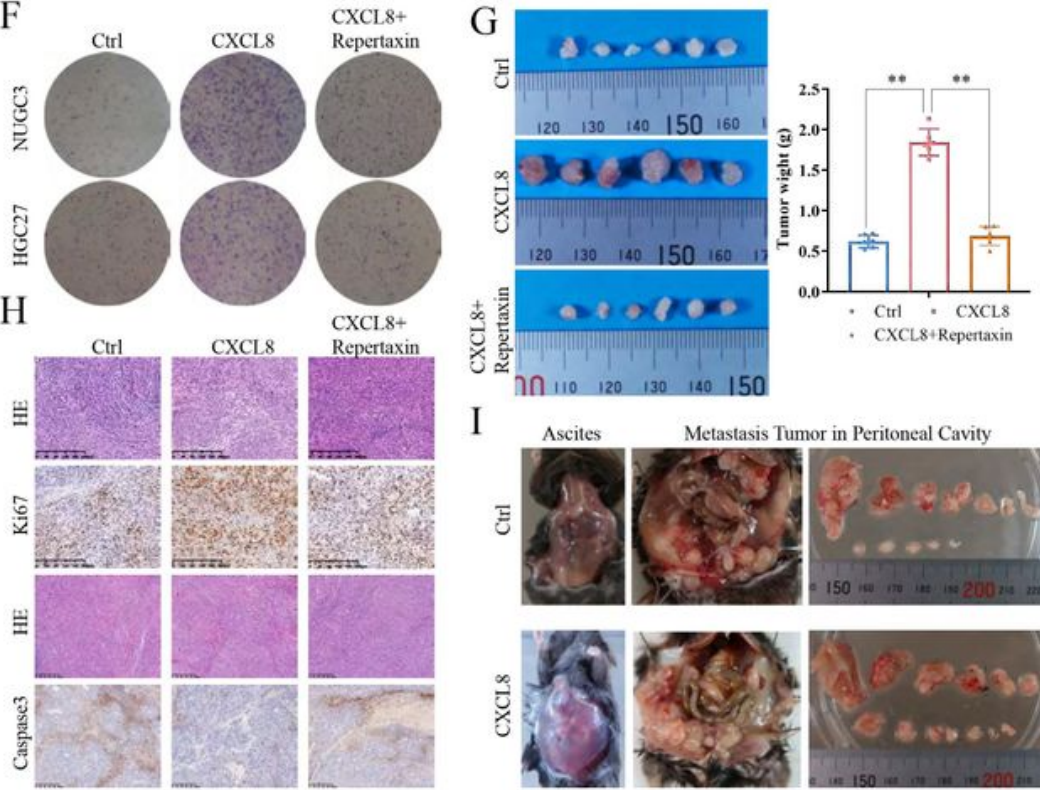

Ascites Metastasis Tumor in Peritoneal Cavity

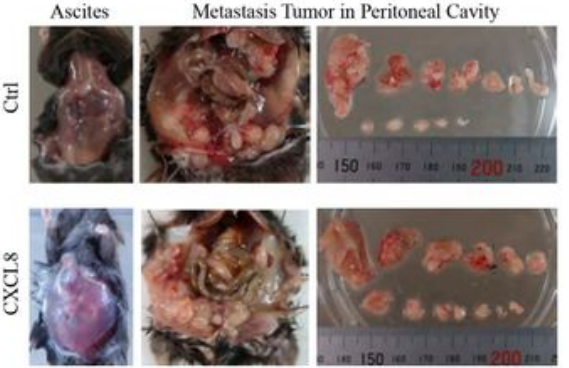

Figure 3

CXCL8-CXCR1/2 deteriorates the malignant phenotype of GC

A. Migration patterns of GC cells on Matrigel compared to cells cultured with CXCL8 and/or CXCL8CXCR1/2 inhibitor repertaxin. 
B. Migration trail of GC cells cultured with $C X C L 8$ or $C X C L 8$ and $C X C L 8-C X C R 1 / 2$ inhibitor.

C. Migration average distances of GC cells in each group.

D. Transwell exhibited the differences in the number of permeable cells in each group.

E, F. CCK-8, and colony formation assay confirmed CXCL 8 could promote GC proliferation which could be inhibited by CXCL8-CXCR1/2 inhibitor.

G. CXCL8 increased the subcutaneous tumor size and weight.

$\mathrm{H}$. IHC representative image of subcutaneous tumors showing that CXCL8 could promote Ki67 and inhibit the expression of Capcase3; the inhibitors could counteract this effect.

I. CXCL8 induced ascites formation and intra- metastasis of GC. 
A

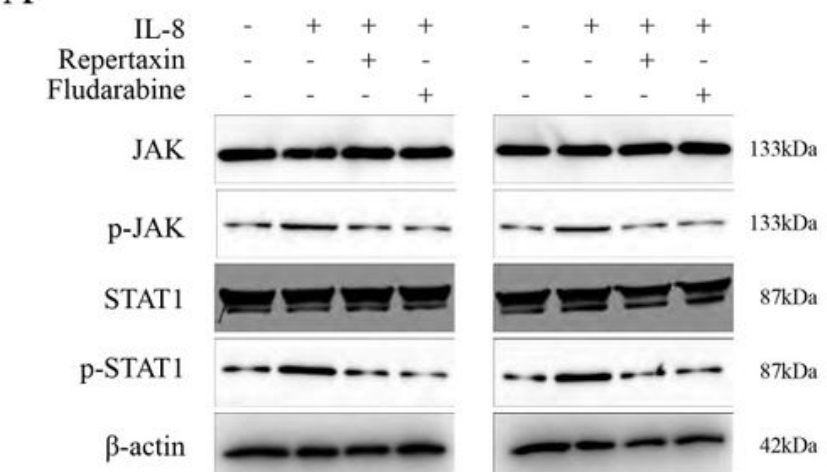

B

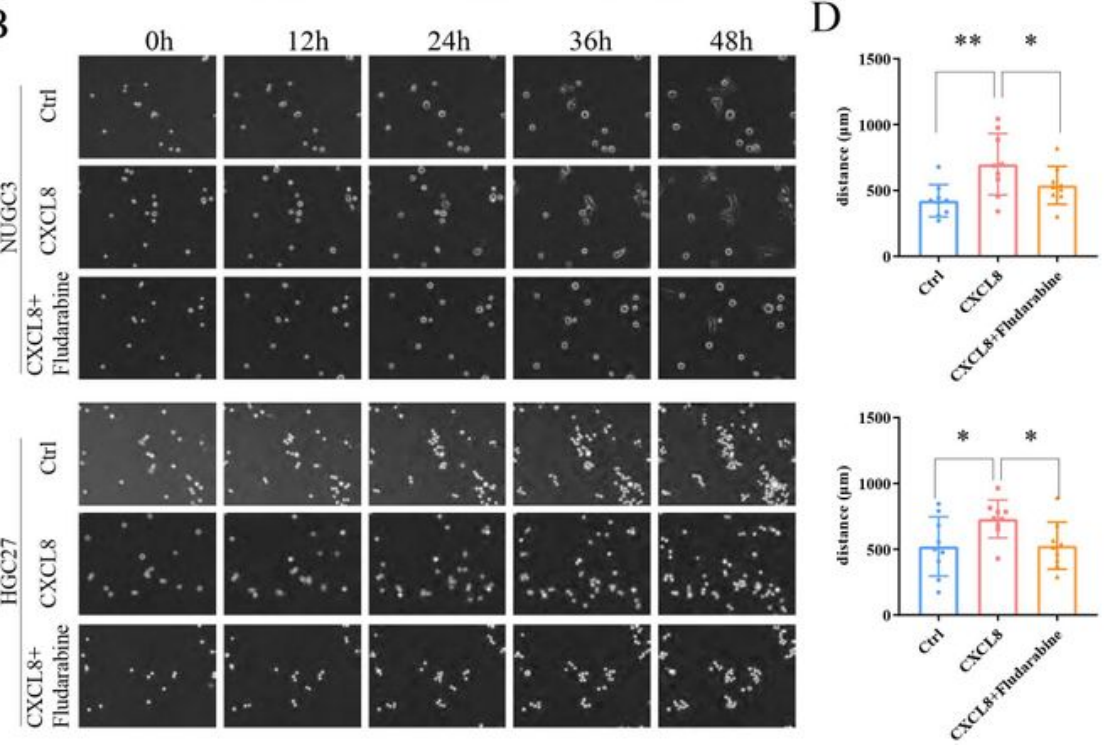

$\mathrm{C}$

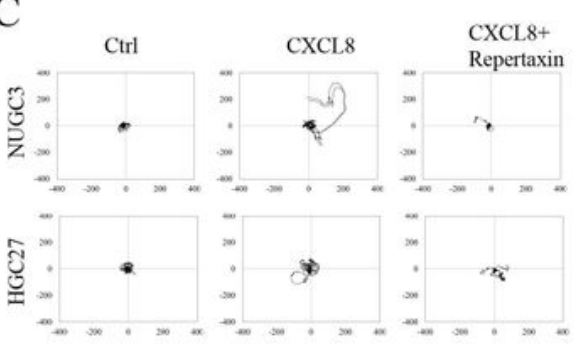

$\mathrm{E}$

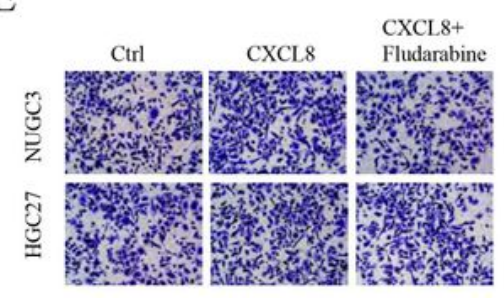

$\mathrm{F}$

G
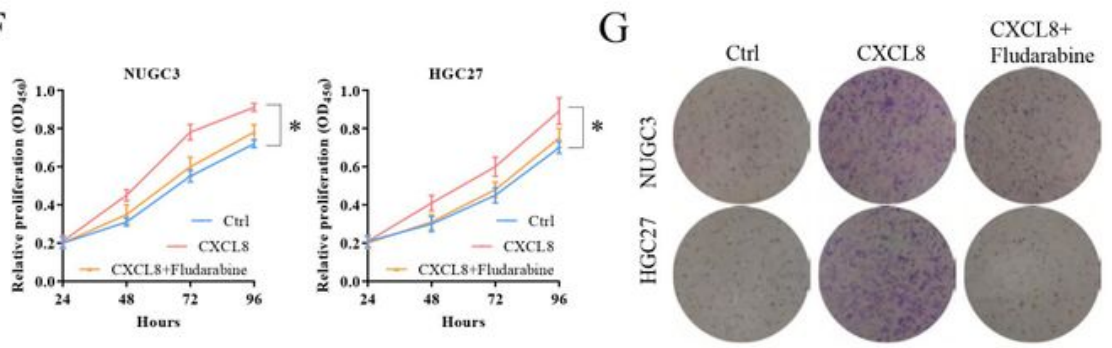

Figure 4

CXCL8-CXCR1/2 activated JAK/STAT1 signal pathway and promoted GC progression.

A. CXCL8 activated the JAK/STAT1 signal pathway. The CXCL8 inhibitor (repertaxin) and STAT1 STAT1 specific inhibitor (Fludarabine) could antagonize the effects of $C X C L 8$. 
B-D. Migration patterns, migration trail, and average traveled distances of GC cells treated with CXCL 8 or CXCL8 and Fludarabine together.

E. Fludarabine limited the GC invasion.

F, G. CCK-8, and colony formation assay revealed that the Fludarabine could resist CXCL8's promotion of GC proliferation

\section{Figure 5}

\section{Transcription factor STAT1 could directly upregulate IL-10 expression.}
A. The shRNA and plasmic regulated STAT1 expression.
B, C. STAT1 regulated HIF-1 a expression at transcription and protein levels.
D, E. STAT1 could also influence $I L-10$ expression at transcription and protein levels.

F, G. The promoter-luciferase reporter assay, showing that STAT1 directly promoted IL-10 expression but not HIF-1a.

$\mathrm{H}$. The interaction of STAT1 with $/ L-10$ shown using ChIP assays with control (IgG) or anti-STAT1 antibody.

I. Deletion and selective mutation analysis identified STAT1-responsive regions in the IL-10 promoter. Luciferase reporter plasmids containing serially truncated or mutated $I L-10$ promoter constructs were cotransfected with OE-STAT1 into NUGC3 cells, and relative luciferase activities were detected. The $-1811 \sim-1821$ sequence was the binding site. 
A

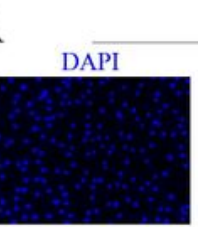

M0 Ctrl
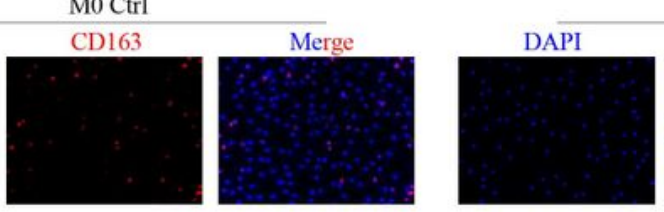

$\mathrm{M} 0+\mathrm{IL}-10$
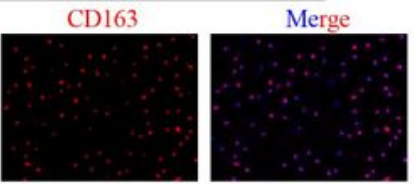

$\mathrm{B}$

Macrophage

C
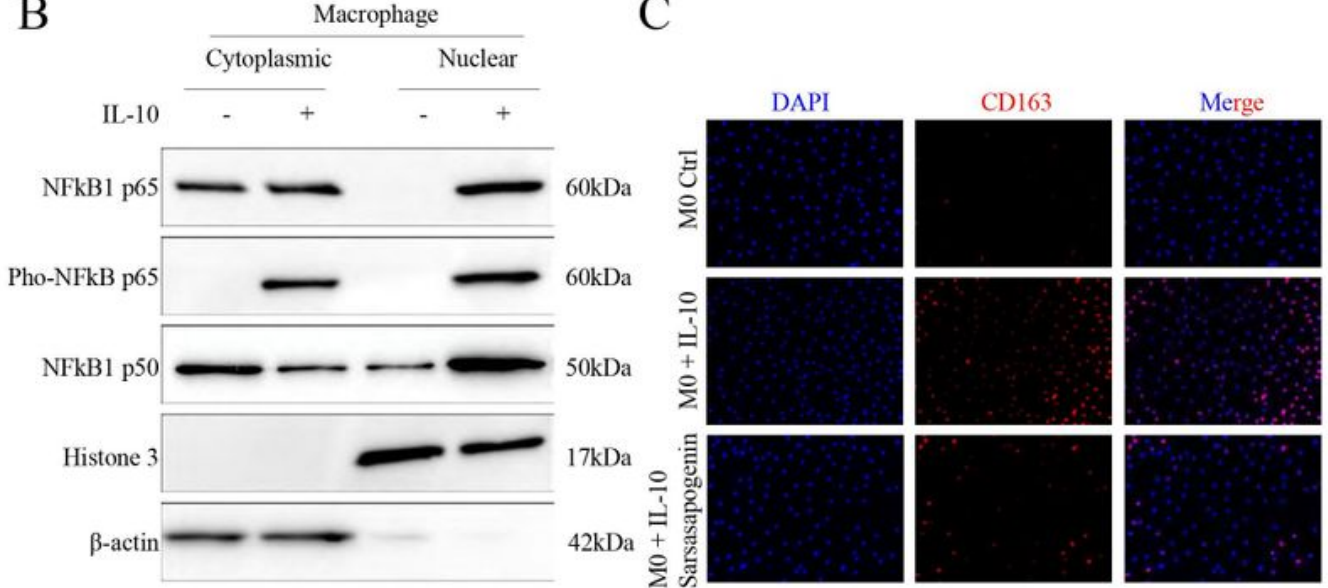

D

E
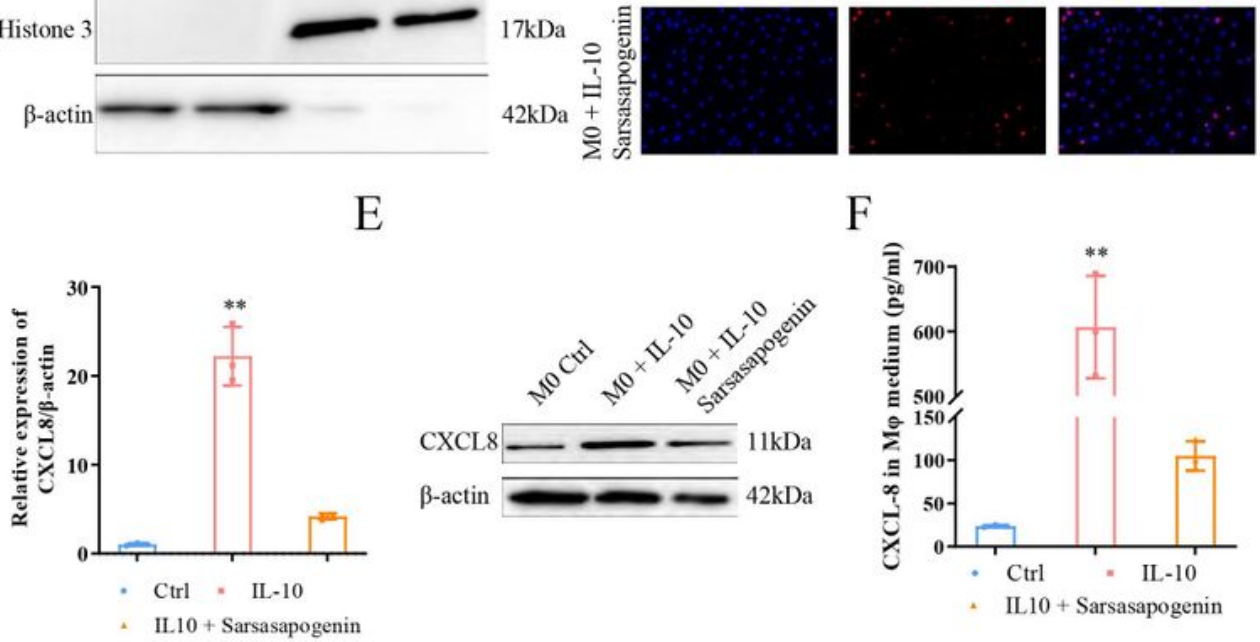

$\mathrm{F}$

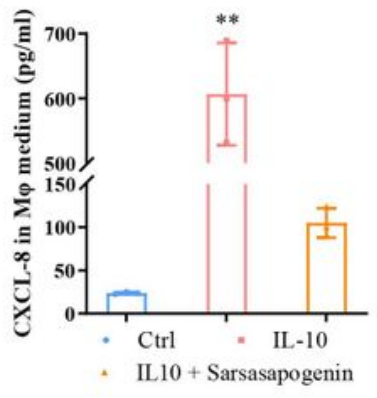

G

$\mathrm{H}$

putative NFKB1 binding site Relative luciferase activity

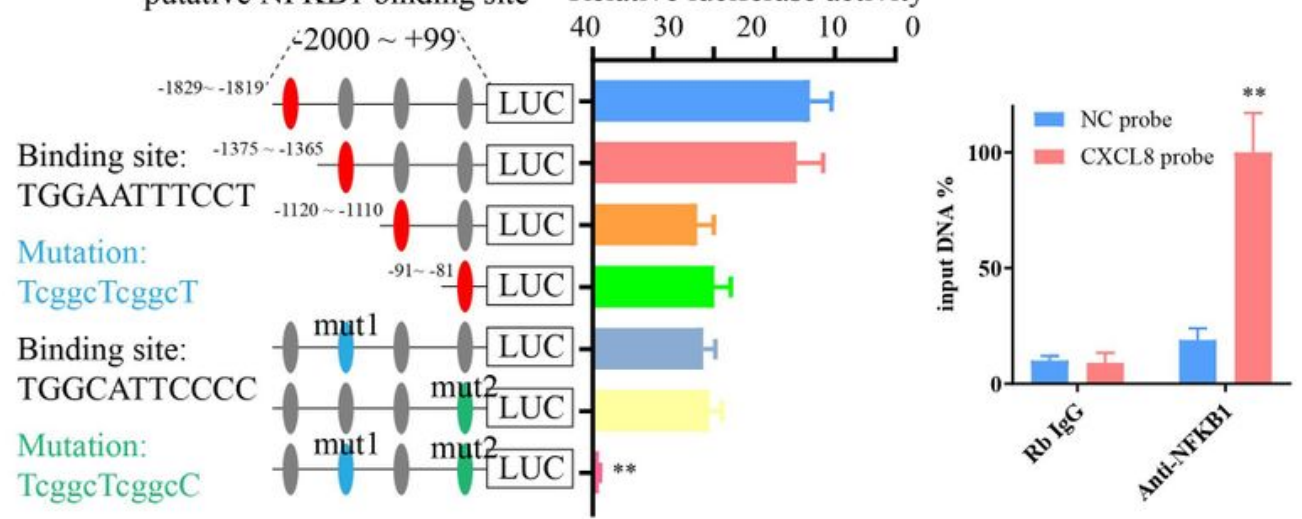

Figure 6

Cytokines IL-10 positive feedback induces macrophage M2 polarization and upregulates macrophagederived CXCL8 expression.

A. IL-10 promoted macrophages CD163 expression and induced M2 polarization. 
B. IL-10 activated NF-KB signaling pathway.

C. The NF-KB pathway inhibitor (Sarsasapogenin) could limit IL-10 induced M2 polarization.

D, E. IL-10 upregulated CXCL8 expression, and it could be prevented by NF-KB pathway inhibitor.

F. IL-10 could promote $C X C L 8$ secretion, and Sarsasapogenin could antagonize the effect of IL- 10 .

G. Deletion and selective mutation analysis recognized the NFKB1-responsive regions in the CXCL8 promoter. Luciferase reporter plasmids containing serially truncated or mutated $C X C L 8$ promoter constructs were co-transfected with $O E-N F K B 1$ into macrophage cells, and relative luciferase activities were detected. The -1829 -1819 and -91 -81 sequences were all the binding sites. 


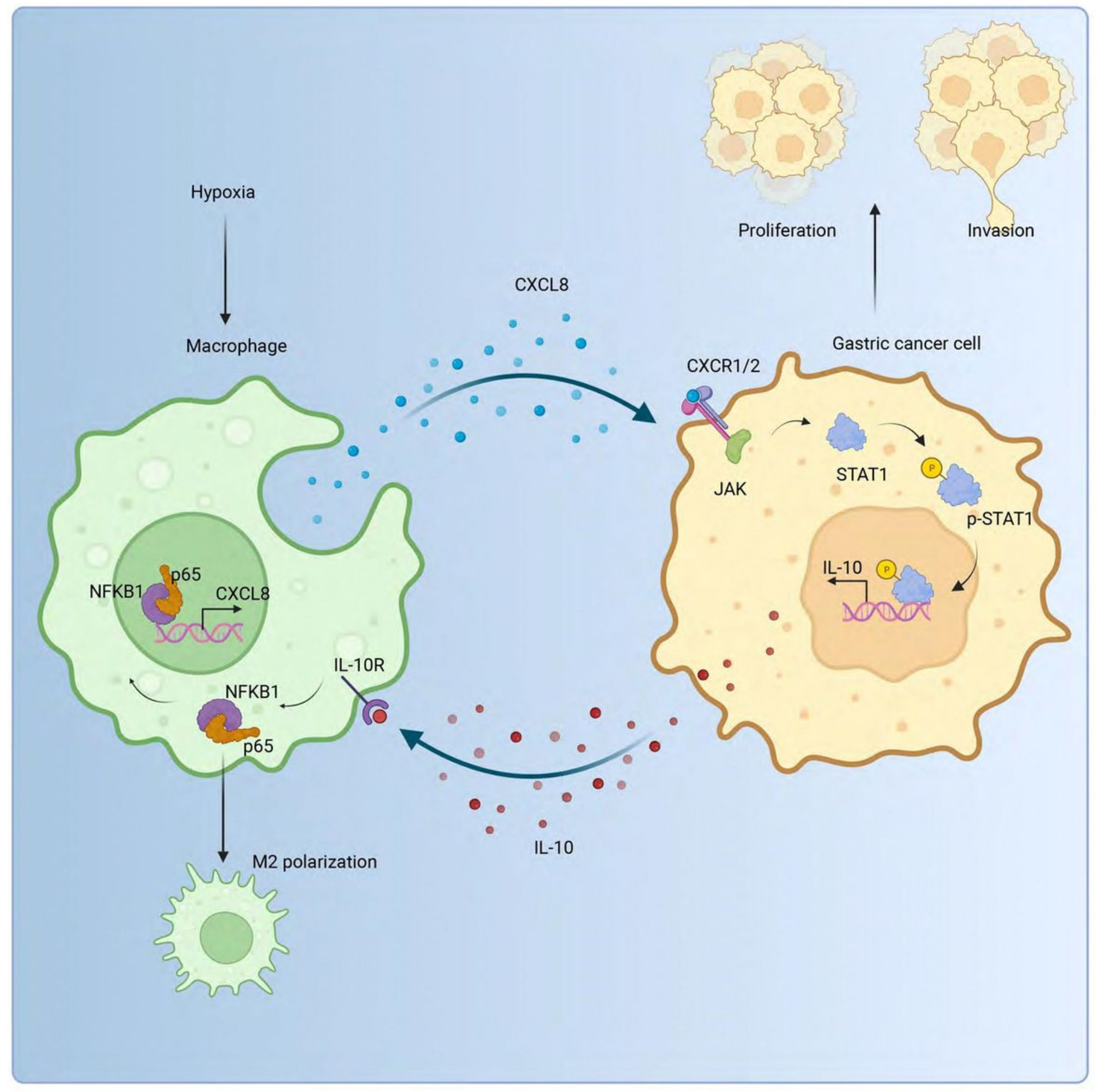

\section{Figure 7}

The mechanism graph of the positive feedback loop between GC cells and TAMs.

Hypoxia-induced macrophage-derived CXCL8 secretion activated CXCL8-CXCR1/2-JAK/STAT1 signaling pathway and deteriorated the GC malignant phenotype. The transcription factor STAT1 could directly promote $I L-10$ expression. The latter activated the macrophage $I L-10 / N F-K B$ signaling pathway and induced M2 polarization. The transcription factor NFKB1 directly upregulated CXCL8 expression. 


\section{Supplementary Files}

This is a list of supplementary files associated with this preprint. Click to download.

- SupTab12.docx

- SupvideoFig3Adown.mp4

- SupvideoFig3Aup.mp4

- SupvideoFig4Adown.mp4

- SupvideoFig4Aup.mp4

- SupplementTable3.csv

- SupplementTable4.xlsx 